Case Report

\title{
A De Novo sSMC (22) Characterized by High-Resolution Chromosome Microarray Analysis in a Chinese Boy with Cat-Eye Syndrome
}

\author{
Jinjie Li $\mathbb{D},{ }^{1}$ Yue Zhang $\mathbb{D}^{1},{ }^{1}$ Yanjun Diao $\mathbb{D},{ }^{1}$ Rui Li $\mathbb{D}^{1},{ }^{1}$ Liqing Jiang $\mathbb{D}^{2},{ }^{2}$ Lei Zhou $\mathbb{D},{ }^{1}$ \\ Jiayun Liu $\left(\mathbb{D},{ }^{1}\right.$ Weixun Duan $\left(\mathbb{D},{ }^{2}\right.$ and Liu Yang $\mathbb{1}^{1}$ \\ ${ }^{1}$ Department of Laboratory Medicine, Xijing Hospital, Air Force Military Medical University, Xi' an 710032, China \\ ${ }^{2}$ Department of Cardiovascular Surgery, Xijing Hospital, Air Force Military Medical University, Xi'an 710032, China \\ Correspondence should be addressed to Weixun Duan; duanweixun@126.com and Liu Yang; zhangzh@fmmu.edu.cn
}

Received 8 May 2020; Revised 18 January 2021; Accepted 14 February 2021; Published 28 February 2021

Academic Editor: Balraj Mittal

Copyright (C) 2021 Jinjie Li et al. This is an open access article distributed under the Creative Commons Attribution License, which permits unrestricted use, distribution, and reproduction in any medium, provided the original work is properly cited.

\begin{abstract}
We report a 15-year-old boy with cat-eye syndrome (CES) without short stature or intellectual disorder. The boy was confirmed by cytogenetic and high-resolution chromosome microarray analysis (CMA). The G-banding karyotype confirmed the de novo of the patient. Also, the CMA result showed $1.76 \mathrm{Mb}$ tetrasomy of proximal 22Q11.1 $\longrightarrow 22 \mathrm{Q} 11.21$ consistent with CES \{arr22q11.1q11.21 $(16,888,899-18,644,241)$ X4\}, a typical small type I CES chromosome. The patient has many of the basic characteristics of CES; however, he is taller than his peers instead of shorter. It is rarely reported in the past since short stature is a common feature of this syndrome. Furthermore, the boy has no intellectual disorder and attends a normal school since he was six-year-old. What bothered him most were recurrent respiratory infections, retromicrognathia, and heart defects.
\end{abstract}

\section{Introduction}

Cat-eye syndrome (OMIM 115470) is a rare chromosomal syndrome associated with a small supernumerary marker chromosome ( $\mathrm{SSMC}$ ), which is derived from duplicated regions of 22pter-22q11.2. The estimated prevalence of CES is $1: 50,000-1: 150,000$ [1]. The syndrome was named "cat eye" due to the typical ophthalmological finding of vertical colobomas in few patients. The most common symptoms are preauricular tags and pits, anal atresia, and coloboma of the iris $[2,3]$. However, clinical features reveal an extreme phenotypic variability, including variable congenital heart defects and kidney and skeletal abnormalities, which may or may not be associated with variable mental deficiency $[2,3]$.

There are about 100 patients reported till now with CES around the world to our knowledge. Here, we report a fifteen-year-old boy with clinical features of CES, confirmed by cytogenetic and high-resolution chromosome microarray analysis (CMA) indicating the breakpoints of the duplication as well as the gene content.

\section{Case Presentation}

When the boy came to our hospital for a bradycardia, he was 15 -year-old. He was the first child of nonconsanguineous and healthy parents. Family history was unremarkable. However, the patient's mother worked at a gas station before the pregnancy, and she moved into a newly renovated house around the pregnancy. Besides, she had been knocked down by a child during the first trimester and treated with utrogestan. The proband was born at 36 weeks gestation with a birth weight of $3400 \mathrm{~g}$. At birth, he was diagnosed with atrial septal and ventricular septal defect resolved by cardiac surgery after one month. He also accepted surgical intervention for subdural effusion and later for inguinal hernia and congenital coloboma of choroid of his right eye. His craniofacial features included long face, broad forehead, downslanting palpebral fissures (Figure 1), strabismus, and bilateral preauricular pits. No hearing loss was noticed. He sometimes ate with eye tears when he swallowed too fast because of the retromicrognathia. Musculoskeletal 


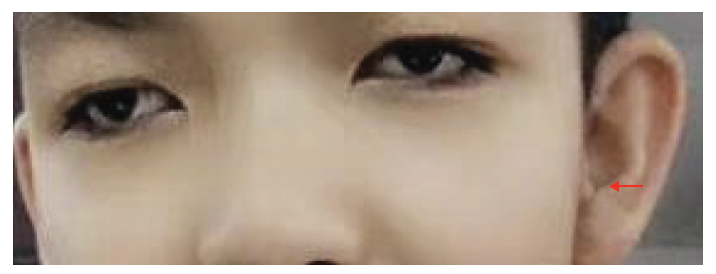

Figure 1: Eyes features of the patient at the age of 15 . Note the downslanting palpebral fissures and strabismus.

abnormalities were the following: kyphoscoliosis, torticollis, longer fingers and toes, rich palm drawing, and pes planus. Especially, he is taller than his peers instead of shorter, his height was $180 \mathrm{~cm}$, and weight was about $45 \mathrm{~kg}$ at the age of 15. And he had no obvious mental disability either. However, he probably had an attention deficit disorder according to his mother's dictation without doing a neuropsychiatric examination. From the neonatal period to the present age, the main health problems of the propositus were recurrent respiratory infections.

\section{Methods}

3.1. Cytogenetic Analyses. The cytogenetic analyses of the patient and his parents with cleft lip and palate were made on G-banded chromosomes obtained from 72-hour lymphocyte cultures.

3.2. High-Resolution Chromosome Microarray Analysis. To ascertain the chromosomal origin and segmental composition of the sSMC, a high-resolution genomic scan using Affymetrix CytoScan $750 \mathrm{~K}$ affay was performed in the patient. Analysis of the arrays was performed using Genotyping Console v4.0 and ChAS v1.2 software.

All samples were taken after an informed consent had been signed.

\section{Results}

4.1. Cytogenetic Analyses. The patient's G-banded karyotype showed a male karyotype 47, XY, +mar. ish idic (22) (q11.2) (20) (Figure 2). However, his father and mother showed a normal male karyotype and a normal female karyotype, respectively (Figure $2 \mathrm{~S}$ ), thus confirming the de novo appearance of the marker chromosome in the proband.

\subsection{High-Resolution Chromosome Microarray Analysis.} The result showed $1.76 \mathrm{Mb}$ tetrasomy of proximal $22 \mathrm{Q} 11.1 \longrightarrow 22 \mathrm{Q} 11.21$ consistent with cat eye syndrome \{arr22q11.1q11.21 $(16,888,899-18,644,241) X 4\}$ (Figure 3). There were 4 copies of 12 genes, including XKR3, IL17RA, CECR1, CECR2, SLC25A18, ATP6V1E1, BID, MICAL3, PEX26, TUBA8, and USP18 (Table 1). The four-copy gains of this region are typically associated with a supernumerary bisatellited marker. However, the present approach was not sufficient to define the parental chromosomal origin of the sSMC.

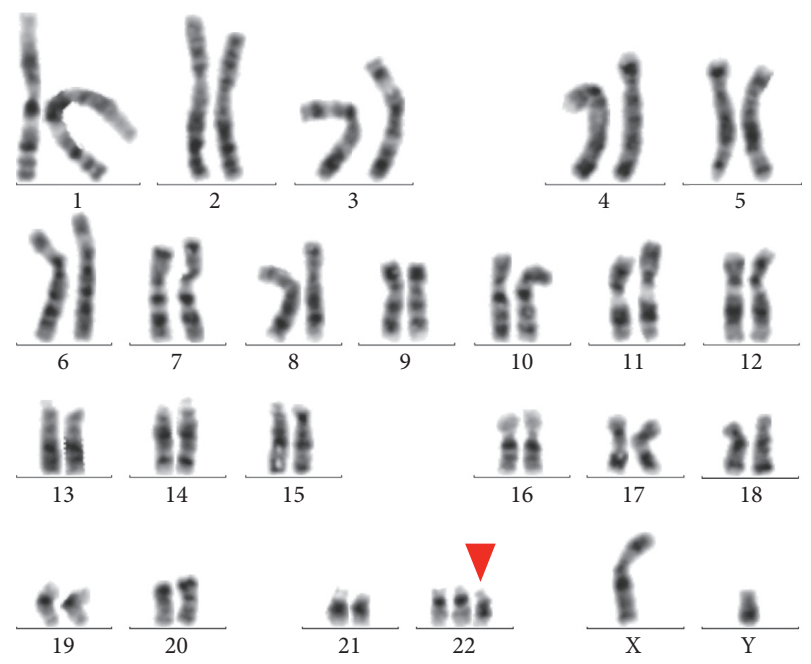

FIgURE 2: G-banding metaphase and karyotype of patient.

\section{Discussion}

Cat-eye syndrome is a rare malformation syndrome which was first reported in 1965 by Schachenmann [5]. The ratio of males to females was nearly $1: 3$ according to the reported cases. We present a 15-year-old boy with many of the basic characteristics of CES as preauricular pits and tags, inferior coloboma of the iris/choroid and retia, retromicrognathia, and downslanting palpebral fissures, but lacking anal atresia or mental retardation [6-9]. In particular, he suffered from congenital heart disease and came to the hospital for a bradycardia.

The patient was referred for genetic evaluation due to the multiple manifestations. Firstly, a routine G-banding karyotype analysis was performed for the proband and his parents. Karyotype analysis of the patient revealed a bisatellited mosaic SMC (Figure 2), and the parents showed normal karyotype in both, thus confirming the de novo of the proband. Then, a chromosome microarray analysis (CMA) was done using Affymetrix CytoScan $750 \mathrm{~K}$ affay for the boy. The result showed $1.76 \mathrm{Mb}$ tetrasomy of proximal $22 \mathrm{Q} 11.1 \longrightarrow 22 \mathrm{Q} 11.21$. The four-copy gains of this region contain 11 genes (Table 1 and Figure 3). Most of them are dosage sensitive, so their function could be affected by extra copies, especially CECR2, the CES-related gene. Comparing to conventional cytogenetic analysis methods, the whole genome microarray scanning technique is of high resolution, high throughput, and high accuracy, which facilitates accurately screening out pathogenic copy number variations and genes and investigating karyotype-phenotype correlation. So the whole genome microarray scanning technique can serve as a useful complement for G-banding to be used in the clinical cytogenetic diagnosis [10].

According to McTaggart et al.'s classification, CES chromosomes fall into two types based on the location of the breakpoints required to generate them. The small type I CES is symmetrical with both breakpoints located with the proximal interval, and the larger type II is either asymmetrical, with one breakpoint located in each of the two intervals, or symmetrical with both breakpoints located in 


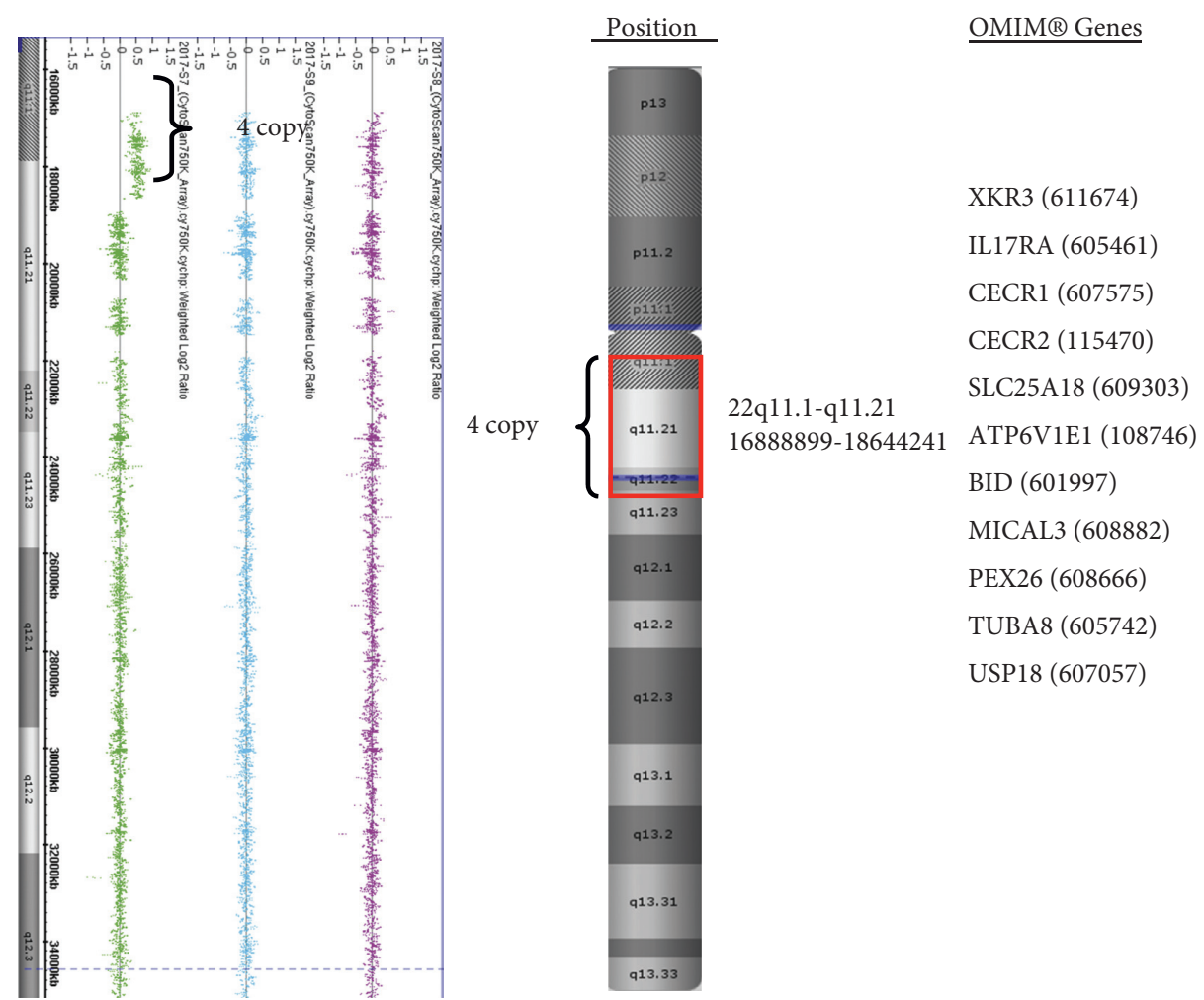

FIGURE 3: Results of high-resolution chromosome microarray analysis.

TABLE 1: Known protein coding genes and their function in the 22q11.1q11.21 (16,888,899-18,644,241), 1.76 Mb duplicated CES region [4].

\begin{tabular}{|c|c|c|c|c|}
\hline Gene & Gene location $^{\mathrm{a}}$ & Name & $\begin{array}{c}\% \\
\mathrm{HI}^{\mathrm{b}}\end{array}$ & Function \\
\hline ATP6V1E1 & $18,074,903-18,111,588$ & $\begin{array}{l}\text { Lysosomal ATPase, H1 transporting, } \\
31 \mathrm{kDa}, \mathrm{V} 1 \text { subunit E1 }\end{array}$ & 48.8 & $\begin{array}{l}\text { V-ATPase responsible for acidifying a variety of } \\
\text { intracellular compartments }\end{array}$ \\
\hline$B I D$ & $18,216,906-18,256,808$ & $\mathrm{BH} 3$ interacting domain death agonist & 89.1 & $\begin{array}{c}\text { Member of the BCL-2 family of cell death } \\
\text { regulators }\end{array}$ \\
\hline CECR1 & $17,660,192-17,690,779$ & CES chromosome region, candidate 1 & 36.7 & $\begin{array}{c}\text { Regulates the concentration of extracellular } \\
\text { adenosine }\end{array}$ \\
\hline CEC & 17,95 & CES chrom & 52.7 & Putative transcri \\
\hline IL17 & 17,56 & Interleukin 1 & 37.5 & nflammatc \\
\hline MICAL3 & $18,270,416-18,507,325$ & $\begin{array}{l}\text { Microtubule-associated monooxygenase } \\
\text { calponin and LIM domain }\end{array}$ & 76.3 & $\begin{array}{l}\text { Cytoplasmic semaphoring/plexin signaling } \\
\text { molecule; expressed in motor neurons }\end{array}$ \\
\hline PEX26 & $18,560,686-$ & Peroxisome biogenesis factor 26 & 98 & Required for protein import into peroxisomes \\
\hline SLC25A18 & $18,043,183-18,073,647$ & $\begin{array}{l}\text { Solute carrier family } 25 \text { (mitochondrial } \\
\text { carrier) }\end{array}$ & 80.9 & $\begin{array}{l}\text { Transport of glutamate across the inner } \\
\text { mitochondrial membrane }\end{array}$ \\
\hline$X K R 3$ & 17,26 & $\begin{array}{l}\text { XK, Kell blood group complex } \\
\text { subunit-related family }\end{array}$ & ND & Component of the XK/Kell blood \\
\hline USP18 & $18,632,758-18,660,162$ & Ubiquitin specific peptidase 18 & ND & $\begin{array}{c}\text { Member of the de-ubiquitinating protease family } \\
\text { of enzymes }\end{array}$ \\
\hline TUBA8 & $18,593,453-18,614,498$ & Alpha tubulin isoform 8 & ND & $\begin{array}{c}\text { Associated with polymicrogyria and optic nerve } \\
\text { hypoplasia }\end{array}$ \\
\hline
\end{tabular}

${ }^{\mathrm{a}}$ University of California Santa Cruz (UCSC) database (http://genome.ucsc.edu/), UCSC Genome Browser, human genome 19/build 37. ${ }^{\mathrm{b}} \mathrm{Haploinsufficiency}$ Score (HI index): high ranks (e.g., 0-10\%) indicate a gene is more likely to exhibit haploinsufficiency and low ranks (e.g., 90-100\%) indicate a gene is more likely to NOT exhibit haploinsufficiency (Huang et al., 2010). CES, cat-eye syndrome; HI, haploinsufficiency score; ND, not determined.

the distal interval $[11,12]$. Our proband has a de novo SMC 22q11.1-q22.2 duplication of $1.76 \mathrm{Mb}$, containing two copies of the CECR rendering the patient tetrasomic for this region. The breakpoints of the duplication, characterized by CMA, begin at the repeated sequences within the chromosome 22 centromere (22q11.1) to the low copy repeat block $\mathrm{A}$ (LCR22-2:16,888,899-18,644,241, hg19) (22q11.21), indicating this to be a small type I CES chromosome [13-15].

CES syndrome shows phenotypic variability, and correlation of clinical severity has neither been found with the 
size of the marker chromosome nor with the extent of mosaicism in mosaic CES. Our patient manifested two of the three typical characteristics:an iris coloboma and ear anomalies. He does not suffer the anal malformation. Only $41.6 \%$ of the reported more than 100 CES patients presented with all the three main characteristics features. The boy in this paper now lives almost a normal life, and he is good at playing drums actually. However, he should pay attention to the recurrent respiratory infections, retromicrognathia, and heart defects, especially the retromicrognathia, which affects his dysphagia.

\section{Conclusion}

Here, we reported a CES case who had a de novo SMC 22q11.1-q22.2 duplication of $1.76 \mathrm{Mb}$ by the whole genome microarray scanning technique. The differences from other cases were that the patient had no obvious mental disability, and he was taller than his peers instead of shorter. It has enriched the phenotypes of the reported cases.

\section{Consent}

Written consent was obtained from the mother of the patient.

\section{Conflicts of Interest}

The authors declare that they have no conflicts of interest.

\section{Acknowledgments}

The authors are grateful to the patient and his parents for their kind contribution to this report. The authors would like to thank the medical teams and technicians whose work supports the clinic.

\section{Supplementary Materials}

The Supplementary Materials contain the G-banding metaphase and karyotype of patient's parents. (Supplementary Materials)

\section{References}

[1] T. N. Win, S. Roberts, and D. Laws, "Duane syndrome associated with the Cat Eye syndrome: a case report," Eye, vol. 21, no. 2, pp. 289-291, 2007.

[2] P. R. Rosias, "Phenotypic variability of the cat eye syndrome. Case report and review of the literature," Genetic Counseling, vol. 12, pp. 273-282, 2001.

[3] M. J. Berends, G. Tan-Sindhunata, B. Leegte, and A. J. van Essen, "Phenotypic variability of Cat-Eye syndrome," Genetic Counseling, vol. 12, pp. 23-34, 2001.

[4] M. Tzetis, K. Stefanaki, A. Syrmou et al., "An unusual case of cat-eye syndrome phenotype and extragonadal mature teratoma: review of the literature," Birth Defects Research Part A: Clinical and Molecular Teratology, vol. 94, no. 7, pp. 561-566, 2012.

[5] G. Schachenmann, W. Schmid, M. Fraccaro et al., "Chromosomes in coloboma and anal atresia," The Lancet, vol. 286, no. 7406, p. 290, 1965.
[6] R. E. Ensenauer, A. Adeyinka, H. C. Flynn et al., "Microduplication 22q11.2, an emerging syndrome: clinical, cytogenetic, and molecular analysis of thirteen patients," The American Journal of Human Genetics, vol. 73, no. 5, pp. 1027-1040, 2003.

[7] T. M. Denavit, V. Malan, C. Grillon et al., "A new case of a severe clinical phenotype of the cat-eye syndrome," Genetic Counseling (Geneva, Switzerland), vol. 15, no. 4, pp. 443-448, 2004.

[8] M. Gentile, S. De Sanctis, F. Cariola et al., "FISH approach to determine cat eye syndrome chromosome breakpoints of a patient with cat eye syndrome type II," European Journal of Medical Genetics, vol. 48, no. 1, pp. 33-39, 2005.

[9] M.-F. Portnoï, "Microduplication 22q11.2: a new chromosomal syndrome," European Journal of Medical Genetics, vol. 52, no. 2-3, pp. 88-93, 2009.

[10] Z. Zheng, R.-e. Yao, J. Geng et al., "A unique combination of 17pter trisomy and 21qter monosomy in a boy with developmental delay, severe intellectual disability, growth retardation and dysmorphisms," Gene, vol. 516, no. 2, pp. 301-306, 2013.

[11] K. E. McTaggart, M. L. Budarf, D. A. Driscoll, B. S. Emanuel, P. Ferreira, and H. E. McDermid, "Cat eye syndrome chromosome breakpoint clustering: identification of two intervals also associated with 22q11 deletion syndrome breakpoints," Cytogenetic and Genome Research, vol. 81, no. 3-4, pp. 222-228, 1998.

[12] O. Bartsch, S. Rasi, K. Hoffmann, and N. Blin, "FISH of supernumerary marker chromosomes (SMCs) identifies six diagnostically relevant intervals on chromosome $22 \mathrm{q}$ and a novel type of bisatellited SMC (22)," European Journal of Human Genetics, vol. 13, no. 5, pp. 592-598, 2005.

[13] T. K. Footz, "Analysis of the cat eye syndrome critical region in humans and the region of conserved synteny in mice: a search for candidate genes at or near the human chromosome 22 pericentromere," Genome Research, vol. 11, no. 6, pp. 1053-1070, 2001.

[14] M. Babcock, S. Yatsenko, J. Hopkins et al., "Hominoid lineage specific amplification of low-copy repeats on $22 \mathrm{q} 11.2$ (LCR22s) associated with velo-cardio-facial/digeorge syndrome," Human Molecular Genetics, vol. 16, no. 21, pp. 2560-2571, 2007.

[15] B. S. Emanuel, "Molecular mechanisms and diagnosis of chromosome 22q11.2 rearrangements," Developmental Disabilities Research Reviews, vol. 14, no. 1, pp. 11-18, 2008. 